\title{
Short- and mid-term multidisciplinary outcomes of newborns exposed to SARS-CoV-2 in utero or during the perinatal period: preliminary findings
}

\author{
Danilo Buonsenso 1,2,3 - Simonetta Costa ${ }^{1}$. Lucia Giordano ${ }^{1} \cdot$ Francessca Priolo ${ }^{1}$ - Arianna Turriziani Colonna ${ }^{1}$. \\ Sofia Morini ${ }^{1}$ - Martina Sbarbati ${ }^{1}$. Davide Pata ${ }^{1}$. Anna Acampora ${ }^{1}$. Guido Conti ${ }^{4}$. Fabrizio Crudo ${ }^{4}$. \\ Alessandro Cantiani ${ }^{4}$. Bianca Maria Martina ${ }^{4}$. Giulia Maria Amorelli ${ }^{5,6} \cdot$ Lorenzo Orazi $^{6} \cdot$ Maria Petrianni $^{6}$. \\ Daniela Ricci $^{6}$. Antonio Lanzone ${ }^{1}$. Maurizio Sanguinetti ${ }^{3,7} \cdot$ Paola Cattani $^{3,7}$. Michela Sali ${ }^{3,7}$. Domenico Romeo ${ }^{1}$. \\ Giuseppe Zampino ${ }^{1} \cdot$ Giovanni Vento $^{1} \cdot$ Piero Valentini $^{1}$
}

Received: 5 August 2021 / Revised: 11 November 2021 / Accepted: 12 November 2021 / Published online: 11 January 2022

(c) The Author(s), under exclusive licence to Springer-Verlag GmbH Germany, part of Springer Nature 2021, corrected publication 2022

\begin{abstract}
The long-term outcomes of newborns exposed to SARS-CoV-2 infection in utero or during the first hours of life are still unknown. We performed a single-center, prospective, observational study of newborns born from mothers with microbiologically confirmed SARS-CoV-2 infection in pregnancy or at time of delivery. Infants were offered a multidisciplinary follow-up consisting of nasopharyngeal Polymerase Chain Reaction test at birth and at 48-72 h of life, auxological growth and neurological development, serologic testing, and audiological and ophthalmological assessments. One-hundred ninety-eight mothers and 199 newborns were enrolled. Of the 199 newborns, 171 underwent nasopharyngeal swab, four (2.3\%) and two (1.15\%) children tested positive at birth and 48-72 h of life, respectively. None had SARS-CoV-2 related symptoms. Auxologic and neurologic development were normal in all children during follow-up. Nine out of 59 infants had SARS-CoV-2 IgG at 3 months of life, which was associated with a positive nasopharyngeal swab at birth $(P=0.04)$. Twenty seven out of $143(18.8 \%)$ newborns had pathologic transitory evoked otoacoustic emissions at birth, although 14/27 repeated after 1 month were normal. Audiological evaluation was completed with Auditory Brainstem Response between the third and sixth month of life in 34 children, showing in all normal hearing threshold. The ophthalmological evaluation found retinal vascular anomalies in $3 / 20$ (15\%) children, immature visual acuity in 5/20 (25\%) children, and reduced distance attention in 6/20 cases (30\%).

Conclusions: Our study showed that the neonatal and mid-term multidisciplinary outcomes of newborns exposed to SARSCoV-2 infection in utero or during the first hours of life are mostly positive, with the exception of ophthalmologic findings which, in a preliminary cohort, were abnormal in about $15 \%$ of cases. Further prospective, longitudinal studies are needed to better understand the clinical outcomes of children exposed to SARS-CoV-2 in utero and in the early postnatal life.

\section{What is Known:}

- In utero mother-to-child transmission of SARS-CoV-2 has been documented by several independent studies.

- Neonatal COVID-19 is a systemic disease that can be severe, although rarely.

What is New:

- Newborns exposed in utero to SARS-CoV-2 have mostly a normal auxological, audiological, and neurological development during the first months of life.

- Fundus fluorescein angiography revealed that up to 5\% of newborns exposed in utero to SARS-CoV2 can show retinal and choroidal abnormalities, including peripheral hypofluorescence of the choroid and increased vascular tortuosity.
\end{abstract}

Keywords COVID-19 · Newborns · Pregnant women · Long-term outcomes

Communicated by Daniele De Luca

Giovanni Vento and Piero Valentini are both last authors.

Extended author information available on the last page of the article 


\section{Introduction}

Since the beginning of the pandemic, the management of pregnant women with COVID-19 and how an infection during pregnancy may affect the fetus and newborns have been challenging the international medical community. Several studies [1-3] and systematic metanalyses [4] showed that the vertical transmission is rare, although possible, and that most perinatal infection are asymptomatic or paucisymptomatic.

While available data raise knowledge to neonatal outcomes of newborns born to positive mothers [5, 6], and allowed the scientific community to better manage the mother-newborn couple (allowing rooming-in and breastfeeding in most cases), they also highlight the current knowledge gap about possible mid- to long-term effects of transplacental or perinatal SARS-CoV-2 neonatal infection. In this state of uncertainty, we can look at other relevant congenital infections to hypothesize how to best follow-up babies born from SARS-CoV-2-infected mothers [7].

During the last decade, we learnt that congenital cytomegalovirus infection, even when newborns are completely asymptomatic, is associated with a significant degree of sensorineural hearing loss and neuropsychiatric involvement [8]. Recent data from a long-term follow-up of children exposed in utero to a newly identified virus, the Zika virus, showed clinical sequelae, even in those children that were not vertically infected [9], further highlighting the importance of establishing a comprehensive follow-up of children exposed to viruses in utero.

SARS-CoV-2 has now a well-established neurotropism that may inflict a wide spectrum of neuropathic effects, potentially including effects on hearing [10]. Neurologic invasion through blood or retrograde neuronal route has been confirmed in the infection of other coronaviruses and the identified receptor for SARS-CoV-2 is present in the nervous system [11]. Focusing on the hearing, a study on 20 asymptomatic adult patients demonstrated that COVID19 infection could have deleterious effects on cochlear hair cell function [12]. A study conducted in Turkey concluded that non-specific symptoms such as sudden sensorineural hearing loss could be the only sign to recognize a COVID19 case [13].

These findings suggest that SARS-CoV-2 may inflict neurosensory damage in exposed fetuses and newborns. Moreover, Lu-Culligan et al. found that the maternal-fetal interface of SARS-CoV-2-infected women exhibited robust immune responses, including increased activation of natural killer (NK) and T cells and increased expression of interferon-related genes [14]. This inflamed placental environment can potentially expose the fetus to a pro-inflammatory background which, in turn, can lead to pathological sequelae, particularly in those organs susceptible to microvascular damages, including the eye.
For these reasons, we hypothesized that both direct viral infection and inflammatory responses during SARSCoV-2 infection in pregnancy may lead to the development of mid- to long-term sequelae in newborns exposed to the SARS-CoV-2 infection. Therefore, we planned a prospective, longitudinal assessment of newborns born from mothers with SARS-CoV-2 infection in pregnancy or at the time of delivery, in order to better define the long-term sequelae of these newborns.

\section{Materials and methods}

\section{Study cohort and data sources}

This is a prospective, observational cohort study of all newborns delivered by women with a confirmed diagnosis of SARS-CoV-2 infection by a molecular Polymerase Chain Reaction (PCR) test performed on nasopharyngeal swab during pregnancy or at the time of delivery, assessed at Fondazione Policlinico Universitario A. Gemelli IRCCS of Rome from March 1, 2020 to April 30, 2021. Our hospital is a Regional Referral Center for pregnant women with proven or suspected SARS-CoV-2 infection. The study was approved by the Ethics Committee of the Fondazione Policlinico Universitario A. Gemelli IRCCS, Rome, Italy (ID 3104). All patients' caregivers provided consent to participate to the study (MOD.PRO.122.001, version 1.1 FPG).

\section{Inclusion criteria}

For this study, we included all newborns or fetuses:

- That were delivered from pregnant women with a documented SARS-CoV-2 infection during any period of pregnancy, including time of delivery;

- Whose parents agreed to enroll a multidisciplinary follow-up detailed below; and

- Had written informed consent to participate from a legal guardian.

\section{Exclusion criteria}

- Newborns delivered from mothers with a suspected but undocumented SARS-CoV-2 infection,

- Newborns whose mothers developed symptoms and were diagnosed with SARS-CoV-2 infection after delivery, and

- Newborns whose parents did not provide written informed consent to participate from a legal guardian. 


\section{Interventions}

Newborns fulfilling inclusion criteria were entered a shortand mid-term follow-up which included:

\section{- Neonatal follow-up}

- In order to define if newborns were vertically infected with SARS-CoV-2, newborns underwent SARS-CoV-2 real-time polymerase chain reaction on nasopharyngeal swab at birth and between 48 and $72 \mathrm{~h}$ of life.

\section{- General pediatric and auxologic follow-up}

- Assessment of weight gain, growth, and head circumference at 3-6-9-12 months of life, using the WHO growth charts.

- Pediatric neurological examination was performed at birth and at 3-6-9-12 months of life. We identified four transition periods $(3,6,9-10,12-14$ months of life) to assess postural development. If these stages were normally achieved, the neurological examination was considered normal. Details on the definition of normal or abnormal neurological outcomes are provided in the supplementary material.

\section{- Infectious disease follow-up}

- Following gained experience from other infectious diseases with potential of mother-to-child transmission (e.g., syphilis and toxoplasmosis), in the assumption that we do not know yet the perfect method of confirmation/exclusion of the infection in the newborn/infant, we enrolled newborns from mother with proven SARS-CoV-2 infection to a serologic assessment of SARS-CoV-2 specific IgG and $\operatorname{IgM} / \operatorname{IgA}$ on peripheral blood samples at the age of 3 months. In case of presence of $\mathrm{IgG}$, families were invited to repeat $\mathrm{IgG}$ dosage every 3 months until 12 months of age to assess if there was clearance of antibodies (meaning maternal $\mathrm{IgG}$ rather than self-production). The CE certified version of the Vircell COVID-19 ELISA antibody kit (Vircell Spain S.L.U., Granada, Spain) was used according to manufacturer's recommendation (https://en.virce 11.com/products/covid-19-elisa/), with a cut-off of $>1$ $\mathrm{UI} / \mathrm{l}$ for positivity. The kit has a sensitivity of $85 \%$ and specificity of $98 \%$ for antibody detection.

\section{- Audiological follow-up}

- The audiological protocol includes the performance of transient evoked otoacoustic emissions (TEOAEs), sounds generated by the outer hair cells within the cochlea in response to auditory stimuli. This test is offered at birth, unless for logistic problems related to the pandemic some could not perform it at birth and were scheduled a follow-up appointment after discharge. Also, regardless the result of neonatal TEOAEs, families were offered to perform the Auditory Brainstem Response (ABR) from the age of 3 months, according to local availability of human and equipment resources during the pandemic. We decided to offer this opportunity to be sure to detect late onset sensorineural hearing loss as documented for CMV infection. Both tests are interpreted according to international guidelines $[15,16]$, and further details are provided in the Supplementary Material. The ABR checks on the function of central nervous system auditory pathways. The following auditory thresholds were considered: normal if $\leq 20 \mathrm{~dB}$ and hypoacusia (unilateral or bilateral) if $\geq 20 \mathrm{~dB}$ (mild 21-40 dB, average 41-70 dB, severe 71-90 dB, deep $>90 \mathrm{~dB}$ ). In case of hypoacusia the child was invited to perform a new ABR test at 6 months of life, in order to confirm or exclude the diagnosis of sensorineural hearing loss.

\section{- Ophthalmologic follow-up}

- The ophthalmological assessment of all infants included in the study was conducted in the Pediatric Retina Department of the Catholic University of the Sacred Heart, Rome. All infants born to COVID19-positive mothers were offered further examination. Only infants whose parents gave informed consent for the examination, and were cooperative enough to obtain good quality images/functional results, were included in the final analysis. All enrolled infants were imaged between 3 and 7 months of age. Parents were educated about the use of oral fluorescein and informed consent was obtained before the procedure. All infants were dilated using tropicamide $1 \%$ drops instilled at 10-min intervals approximately $30 \mathrm{~min}$ before the imaging session. A $20 \%$ fluorescein sodium solution dosage was calculated according to recommendations $(7.5 \mathrm{mg} / \mathrm{kg}$ under 18 years old) [17], mixed with infant formula milk, and given to the patient 10 to $20 \mathrm{~min}$ before the imaging procedure. Non-contact high-resolution ultra-widefield scanning laser ophthalmoscope (Optos California, Optos PLC, Dunfermline) was used to obtain fast retinal angiographic images, following application of topical anesthetic drops (oxybuprocaine chlorhydrate $0.4 \%$ ). The infant was held up to the imaging lens in the "flying baby" position by the examiner supporting the head of the baby with one hand and the other 
supporting the rest of the body. No lid speculum nor sedation was necessary during the procedure. Medical personnel was at all times present to monitor the vital signs of the infant. All infants also underwent imaging using the Envisu 2300 portable hand-held SD-OCT (Bioptigen Incorporated, Durham, NC). Scans were obtained following parameters provided by Maldonado et al. [18]. The scan size was $8 \times 8 \mathrm{~mm}$ and scan density was 1000 A scans/100 B scans. Images were included only if the entire foveal region was visible and image quality was adequate to segment inner (IRL) and outer (ORL) retinal layers.

\section{- Behavioral assessment of visual function}

- This included an assessment of various aspects of visual function such as ability to fix and follow, saccades, acuity, visual fields, attention at distance, and contrast sensitivity [19-22]. Ocular motility was also observed. More specifically presence of nystagmus, presence of horizontal and/or vertical deviations of the eyes, and anomalous head posture or abnormal ocular movements were recorded. Particularly, we assessed the following functions, which are detailed in the Supplementary Material: Ability to fix, Ability to track, Saccades, Visual Acuity, Attention at distance, Binocular visual fields, and Contrast sensitivity. A total score was calculated by adding the score recorded for every single item: score of 0 was given for normal results and a score of 1 for abnormal findings. A total score $\geq 2$ was considered abnormal.

\section{Primary objective}

The objective was to assess short- (neonatal period) and mid-term (first year of life) multidisciplinary outcomes (auxological, neurological, serological, audiological, and ophthalmological) of infants born to a mother with SARSCoV-2 infection during pregnancy or at time of delivery.

Since the main objective was to assess the outcomes of every newborn exposed to the virus during pregnancy, also newborns that were not tested before were included at birth.

\section{Secondary objective}

- Second objective was to assess if maternal symptoms during COVID-19, degree of inflammatory markers, and trimester of maternal infection correlated with neonatal outcomes.

\section{Statistical analysis}

Data were analyzed by creating descriptive tables. Numerical continuous variables, such as mother's age at the time of delivery and $Z$-score for neonatal weight, height, and head circumference, were expressed as median and inter-quartile range (IQR). Categorical variables (such as pregnancy trimester in which the mother got infected, symptoms, therapies, PCR levels on maternal blood samples, D-dimer and lymphocytes, type of birth, neonatal sex, result of neonatal nasopharyngeal swab and antibody research, results of audiological and ophthalmological evaluations) were expressed through absolute frequency and percentages.

The possible association between maternal age at the time of the delivery (that was divided in 4 categories $<20$ years old, 20-30 years old, 30-40 years old, $>40$ years old), trimester in which the mother got infected, symptoms, PCR levels $(<5 \mathrm{mg} / \mathrm{dl}$, $5-49.9 \mathrm{mg} / \mathrm{dl},>50 \mathrm{mg} / \mathrm{dl})$, D-dimer $(<500 \mathrm{mg} / \mathrm{dl}, 500-1499 \mathrm{mg} /$ $\mathrm{dl}, \geq 1500 \mathrm{mg} / \mathrm{dl}$ ), lymphocytes (<1.5 U/ml, $1.5-3 \mathrm{U} / \mathrm{ml},>3 \mathrm{U} /$ $\mathrm{ml})$, medical treatment used for COVID-19, gestational age (<37 weeks, $\geq 37$ weeks), type of birth (vaginal delivery or cesarean section), and the results of TEOAES was analyzed through univariate analysis by using chi-squared test. If expected frequencies were inferior to 5, Fisher exact test was used. A possible association between those variables and $Z$-score for auxological data was explored through the Mann-Whitney rank test.

To assess the $Z$-score for auxological data trend both univariate and multivariate analyses were used, through Generalized Estimating Equation that takes into account the longitudinal nature of the data. Beta coefficients express the $Z$-score variation for each follow-up compared to T0. Together with beta coefficients, confidence interval (CI) at $95 \%$ was estimated.

If CI 95\% includes "1," auxological data trends are not statistically significant. Clinical significance was considered, together with statistical significance.

Data trends were depicted by the construction of boxplots for 5 different times of follow-up. A boxplot is constructed of two parts, a box and a set of whiskers. The lowest point is the minimum of the data set and the highest point is the maximum of the data set. The box is drawn from first quartile to third quartile with a horizontal line drawn in the middle to denote the median. Any data not included between the whiskers are plotted as an outlier with a dot.

Given that some epidemiological aspects of this health emergency are little known or unknown, we refer to the positivity to SARS-Cov-2 of the 3 infants out of 33 (equal to $9 \%$ ), reported by Zeng et al. [23] for the calculation of the sample size. Based on this assumption, to detect the longterm effects of women with Sars-Cov-2 infection in pregnancy, a sample of 119 pregnant women is needed, with a margin of error of $5 \%$ and a confidence interval of $95 \%$. However, this sample size is defined to detect the vertical transmission of the virus. Considering that there are no data about the longer-term outcomes, we could not define a sample and we decided to enroll all patients assessed so far, in order to provide preliminary data that will be useful for defining future study samples. 


\section{Results}

\section{Study population}

From March 2020 to April 2021, 198 mothers and 199 newborns were enrolled (one woman had a twin pregnancy). The majority of women delivered from September 2020 to April 30 (Fig. 1), corresponding to the second and third Italian waves, since during the first wave Rome was not as severely affected as Northern Italy. The enrolled mothers had an average age of 33 years (IQR 28-37 years) at the time of delivery. Ninety-three percent contracted SARSCoV-2 infection in the third trimester of pregnancy (112 of them having active infection at the time of delivery), six women $(3.5 \%)$ contracted the infection in the first trimester and $6(3.5 \%)$ in the second trimester. Of the mothers, 34.4\% had symptomatic disease. Further clinical and laboratory details about maternal SARS-CoV-2 infection are described in Table 1. The effect of trimester of infection, inflammatory markers (including D-dimers), and treatments received on gestational age at delivery and type of delivery are reported in the Supplementary Tables S1 and S2, respectively. In general, none of the mentioned factors affected gestational age and type of delivery, except for anticoagulants used during pregnancy, which were significantly associated with a higher probability of cesarean section $(P<0.0001)$.

\section{Neonatal outcomes}

One-hundred ninety-nine newborns were delivered (Fig. 1), $129(64.8 \%)$ by vaginal delivery and $58(29.2 \%)$ by caesarean section (Table 2). Of the vaginal deliveries, four required the use of a suction cup, while among the caesarean sections, six were performed urgently. Among them, 1 due to maternal eclampsia.

The median birth weight of the children was $3242.5 \mathrm{~g}$ (IQR 2950-3550), Z-score + 0.08; the median length at birth was $50 \mathrm{~cm}$ (IQR 49-50), Z-score + 0.49; and the median head circumference was 34 (IQR 34-35), Z-score + 0.55. Auxological parameters at birth were not affected by trimester of infection, type of SARS-CoV-2 infection (symptomatic vs asymptomatic), inflammatory markers, and D-dimers and treatment received by the mother (Supplementary table S3).

At birth, 171 of the 199 newborns considered were subjected to nasopharyngeal swab at birth and at $48-72 \mathrm{~h}$ of life. One-hundred sixty seven (97.7\%) were negative at birth. Four children tested positive $(2.3 \%)$ at the first swab performed $<24 \mathrm{~h}$ after birth. At $48 \mathrm{~h}$ of age, 2 of the 4 babies who tested positive at birth were negative (indeterminate infection), while the other two cases had a confirmed positive nasopharyngeal test (possible infection, since it was from a non-sterile sample, according to the WHO definition available at https://www.who.int/publications/i/item/

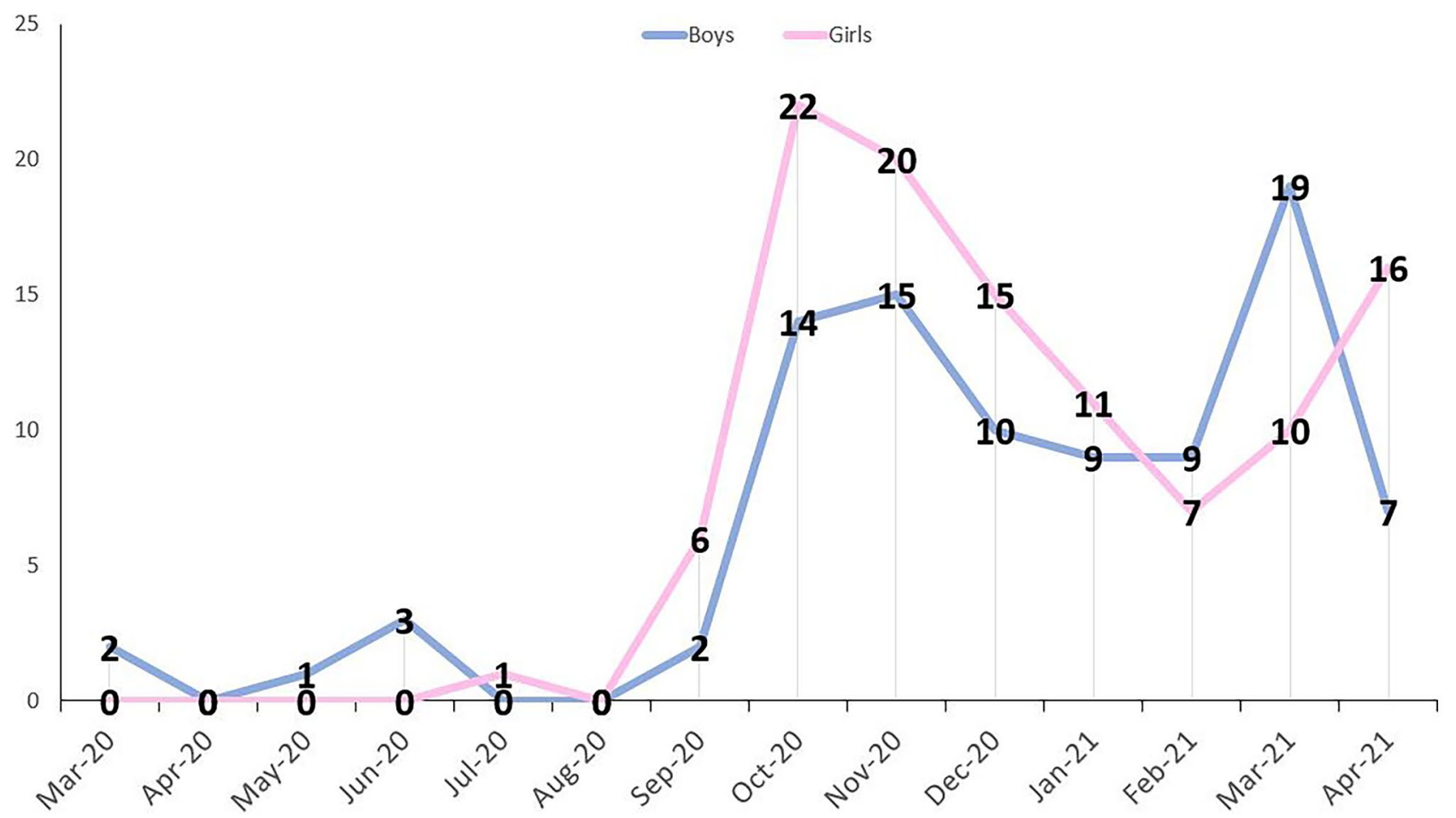

Fig. 1 Distribution of births during the study period 
Table.1 Characteristics of pregnant women enrolled

\begin{tabular}{|c|c|}
\hline $\begin{array}{l}\text { Characteristics of enrolled pregnant } \\
\text { women }\end{array}$ & $\begin{array}{l}\text { All mothers } \\
(\mathrm{N}=198)\end{array}$ \\
\hline Age (median, IQR) & $33(28-37)$ \\
\hline \multicolumn{2}{|l|}{ Period of COVID-19 diagnosis $(n, \%)$} \\
\hline $\begin{array}{l}\text { First trimester } \\
\text { Second } \\
\text { Third }\end{array}$ & $\begin{array}{l}6(3.4) \\
6(3.4) \\
167(93.2)\end{array}$ \\
\hline \multicolumn{2}{|l|}{ Type of infection } \\
\hline $\begin{array}{l}\text { Symptomatic } \\
\text { Asymptomatic }\end{array}$ & $\begin{array}{l}68(34.4) \\
115(57.6)\end{array}$ \\
\hline \multicolumn{2}{|l|}{ Symptoms } \\
\hline $\begin{array}{l}\text { Fever } \\
\text { Rhinitis } \\
\text { Cough } \\
\text { Pharyngodynia } \\
\text { Dyspnea } \\
\text { Gastrointestinal symptoms } \\
\text { Ageusia } \\
\text { Anosmia }\end{array}$ & $\begin{array}{l}36(18.1) \\
14(7.0) \\
21(10.6) \\
6(3.0) \\
10(5.0) \\
2(1.0) \\
24(12.1) \\
25(12.6)\end{array}$ \\
\hline \multicolumn{2}{|l|}{ Therapies } \\
\hline $\begin{array}{l}\text { Any non-invasive respiratory support } \\
\text { Intensive care } \\
\text { Mechanical ventilation } \\
\text { Steroids } \\
\text { Lopinovir-ritonavir } \\
\text { Azythromicine } \\
\text { Remdesivir } \\
\text { Clexane } \\
\text { Idrossiclorochina } \\
\text { FANS } \\
\text { Antibiotics }\end{array}$ & $\begin{array}{l}11(5.53) \\
0 \\
0 \\
0 \\
2(1.0) \\
8(4.0) \\
0 \\
110(55.3) \\
3(1.5) \\
58(29.2) \\
48(24.1)\end{array}$ \\
\hline \multicolumn{2}{|l|}{ Radiologically documented pneumonia } \\
\hline $\begin{array}{l}\text { Yes } \\
\text { No }\end{array}$ & $\begin{array}{l}31(15.6) \\
168(84.4)\end{array}$ \\
\hline \multicolumn{2}{|l|}{ PCR } \\
\hline $\begin{array}{l}<5 \mathrm{ng} / \mathrm{ml} \\
5-50 \mathrm{ng} / \mathrm{ml} \\
>50 \mathrm{ng} / \mathrm{ml}\end{array}$ & $\begin{array}{l}1(0.5) \\
34(17.09) \\
138(69.35)\end{array}$ \\
\hline \multicolumn{2}{|l|}{ Lymphocytes } \\
\hline $\begin{array}{l}<1500 \mathrm{cells} / \mathrm{mmc} \\
1500-3000 \mathrm{cells} / \mathrm{mmc} \\
>3000 \mathrm{cells} / \mathrm{mmc}\end{array}$ & $\begin{array}{l}50(25.13) \\
79(39.70) \\
12(6.03)\end{array}$ \\
\hline \multicolumn{2}{|l|}{ D-dimers } \\
\hline $\begin{array}{l}<500 \mathrm{mg} / \mathrm{dl} \\
500-1500 \mathrm{mg} / \mathrm{dl} \\
>1500 \mathrm{mg} / \mathrm{dl}\end{array}$ & $\begin{array}{l}1(0.5) \\
20(10.05) \\
64(32.16)\end{array}$ \\
\hline
\end{tabular}

WHO-2019-nCoV-mother-to-child-transmission-2021.1). In 28 newborns from mothers with SARS-CoV-2 infection during the first or second trimester, parents did not provide consent for neonatal swab, but accepted to enroll the newborns in the dedicated follow-up.

All children who tested positive for nasopharyngeal swab were asymptomatic for signs or symptoms related to SARSCoV-2 infection.
Table.2 Neonatal outcomes

\begin{tabular}{ll}
\hline Characteristics of neonates & Newborns $(\mathbf{N}=\mathbf{1 9 9})$ \\
\hline Type of delivery & \\
Vaginal delivery & $129(64.82)$ \\
Cesarean section & $58(29.15)$ \\
Type of delivery - specific & \\
Vaginal delivery & $125(62.81)$ \\
Cesarean section & $52(26.13)$ \\
Operative vaginal delivery & $4(2.01)$ \\
Urgent cesarean section & $6(3.02)$ \\
Weight (median, IQR) & $3242.5(2950-3550)$ \\
Z-score & $+0.08(-0.53-+0.82)$ \\
Length (median, IQR) & $50(49-50)$ \\
Z-score & $+0.49(-0.05-+0.96)$ \\
Head circumference (median, IQR) & $34(34-35)$ \\
Z-score & $+0.55(-0.07-+1.19)$ \\
First neonatal swab & \\
Positive & $4(2.0)$ \\
Negative & $167(83.9)$ \\
Second neonatal swab & \\
Positive & $2(1.0)$ \\
Negative & $167(97.7)$ \\
Type of neonatal infection & \\
Symptomatic & \\
Asymptomatic & 0 \\
\hline
\end{tabular}

We found no cases of malformations or syndromes in the cohort of neonates.

\section{Serological outcomes}

So far, the antibody search against SARS-CoV-2 was performed on the serum of 59 patients at 3 months of age (Table 3). Nine patients had positive IgG levels. Neither the trimester of infection nor the type of maternal infection affected the probability of a positive IgG response $(P<0.05)$, while a positive nasopharyngeal swab at birth was more frequently associated with a positive result $(P=0.04)$.

So far, none of the enrolled children developed signs or symptoms suggestive of a postnatal SARS-CoV-2 infection.

\section{General pediatric and neurologic outcome}

At the time of writing of this preliminary study (still ongoing), data on the auxological growth and neurological development were available for 120 children at 1 month of life (T1), 82 at 3 months of life (T2), 40 at 6 months of life (T3), and 20 at 9 months of life (T4). Figure 2 shows boxplots with the trend of the $Z$-scores of weight, height, and head circumference during the different timepoints. $Z$-scores were always within the normal values for age, between values of +1.88 and -1.88 . Growth was not affected by trimester of infection, type of disease, and 
Table.3 Audiologic assessment

\begin{tabular}{|c|c|c|c|}
\hline & \multicolumn{2}{|l|}{ TEOAEs } & \multirow[t]{2}{*}{$P$-value } \\
\hline & normal & pathologic & \\
\hline \multicolumn{4}{|c|}{ Trimester of maternal infection } \\
\hline$I$ & $6(5.8)$ & 0 & 0.426 \\
\hline II & $4(3.9)$ & $2(8.00)$ & \\
\hline III & $94(90.3)$ & $23(92.00)$ & \\
\hline \multicolumn{4}{|c|}{ Symptomatic mother } \\
\hline Yes & $43(39.09)$ & $8(34.78)$ & 0.815 \\
\hline No & $67(60.91)$ & $15(65.22)$ & \\
\hline \multicolumn{4}{|c|}{ Maternal D-dimers } \\
\hline$<500 \mathrm{mg} / \mathrm{dl}$ & $14(26.42)$ & $2(18.18)$ & 0.716 \\
\hline$>500 \mathrm{mg} / \mathrm{dl}$ & $39(73.58)$ & $9(81.82)$ & \\
\hline \multicolumn{4}{|c|}{ Maternal C-reactive protein } \\
\hline$\leq 5 \mathrm{mg} / \mathrm{dl}$ & $24(60.00)$ & $4(50.00)$ & 0.703 \\
\hline$>5 \mathrm{mg} / \mathrm{dl}$ & $16(40.00)$ & $4(50.00)$ & \\
\hline \multicolumn{4}{|c|}{ Mothers received steroids } \\
\hline Yes & $8(6.90)$ & $2(7.69)$ & $>0.999$ \\
\hline No & $108(93.10)$ & $24(92.31)$ & \\
\hline \multicolumn{4}{|c|}{ Mothers received enoxaparine } \\
\hline Yes & $65(56.03)$ & $12(46.15)$ & 0.390 \\
\hline No & $51(43.97)$ & $14(53.85)$ & \\
\hline \multicolumn{4}{|l|}{ Maternal age } \\
\hline$<20$ years & $1(0.92)$ & 0 & 0.203 \\
\hline 20-30 years & $30(27.52)$ & $6(25.00)$ & \\
\hline $30-40$ years & $64(58.72)$ & $18(75.00)$ & \\
\hline$>40$ years & $14(12.84)$ & 0 & \\
\hline \multicolumn{4}{|c|}{ Maternal lymphocyte count } \\
\hline$<1.5$ cells $/ \mathrm{mm}^{3}$ & $30(35.71)$ & $4(22.22)$ & 0.132 \\
\hline $1.5-3$ cells $/ \mathrm{mm}^{3}$ & $52(61.90)$ & $12(66.67)$ & \\
\hline$>3$ cells $/ \mathrm{mm}^{3}$ & $2(2.38)$ & $2(11.11)$ & \\
\hline
\end{tabular}

values of maternal inflammatory markers and $\mathrm{D}$-dimers. Importantly, none of the infants evaluated at each timepoint showed abnormal findings at the neurological assessment.

\section{Audiological outcomes}

One-hundred forty-three newborns underwent TEOAEs at birth, of which 27 were found to be pathological (refer).
Among patients who had normal TEOAEs at birth, six (5.8\%) were born to mothers who had contracted the infection in the first trimester of pregnancy, five $(3.9 \%)$ in the second, and 94 (90.3\%) in the third trimester. However, none of the investigated maternal factors affected the result of TEOAEs at birth (Table 3).

Of the 26 children who had pathological TEOAEs at birth, 14 repeated the examination after 1 month and all were normal. Audiological evaluation was completed with ABR between the third and sixth month of life in 34 children. All children showed a normal hearing threshold $(20 \mathrm{~dB})$.

\section{Ophthalmologic follow-up}

The ophthalmological evaluation included three parts: spectral domain-optical coherence tomography (SD-OCT), ultrawidefield fluorescein angiography (FFA), and behavioral assessment of visual functions. All the 20 enrolled infants were imaged between 3 and 7 months of age. Two were excluded due to poor quality images.

SD-OCT was performed for all 20 infants: no attenuation of ganglion cell layer (GCL), paracentral acute middle maculopathy (PAMM), subretinal fluid, cystoid macular edema (CME), or subfoveal thickening were highlighted. All children presented age-appropriate retinal development.

Twenty children underwent oral FFA (Fig. 2). Good quality images were obtained for all 20 infants (Table 4). Bilateral choroidal perfusion anomalies were highlighted in 3 infants (15\%), showing peripheral hypofluorescence of the choroid. A slight obliteration of the capillary bed was also observed. One of the 20 children presented bilateral vascular tortuosity. This patient also tested negative for nasopharyngeal swab at birth and at $48 \mathrm{~h}$ of life and for serology at the third month of life.

Twenty neurovisual tests were performed on 20 children. The age range was between 2 and 7 months (mean 4.7 months). All children exhibited normal fixation skills and complete tracking skills. Saccadic movements were present in all infants over 3 months of age.
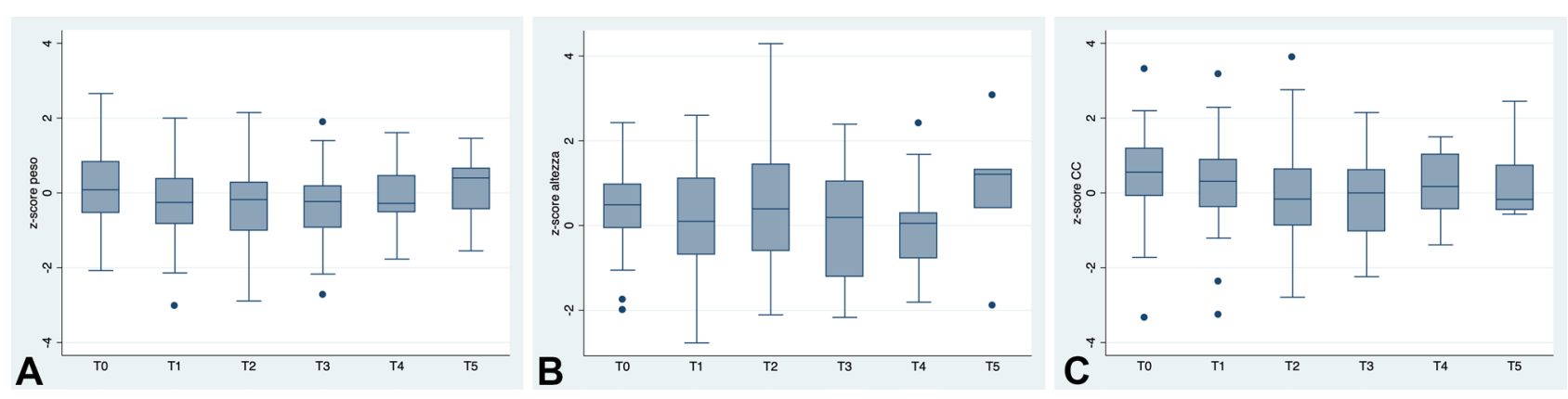

Fig. 2 Auxological growth during different timepoints 


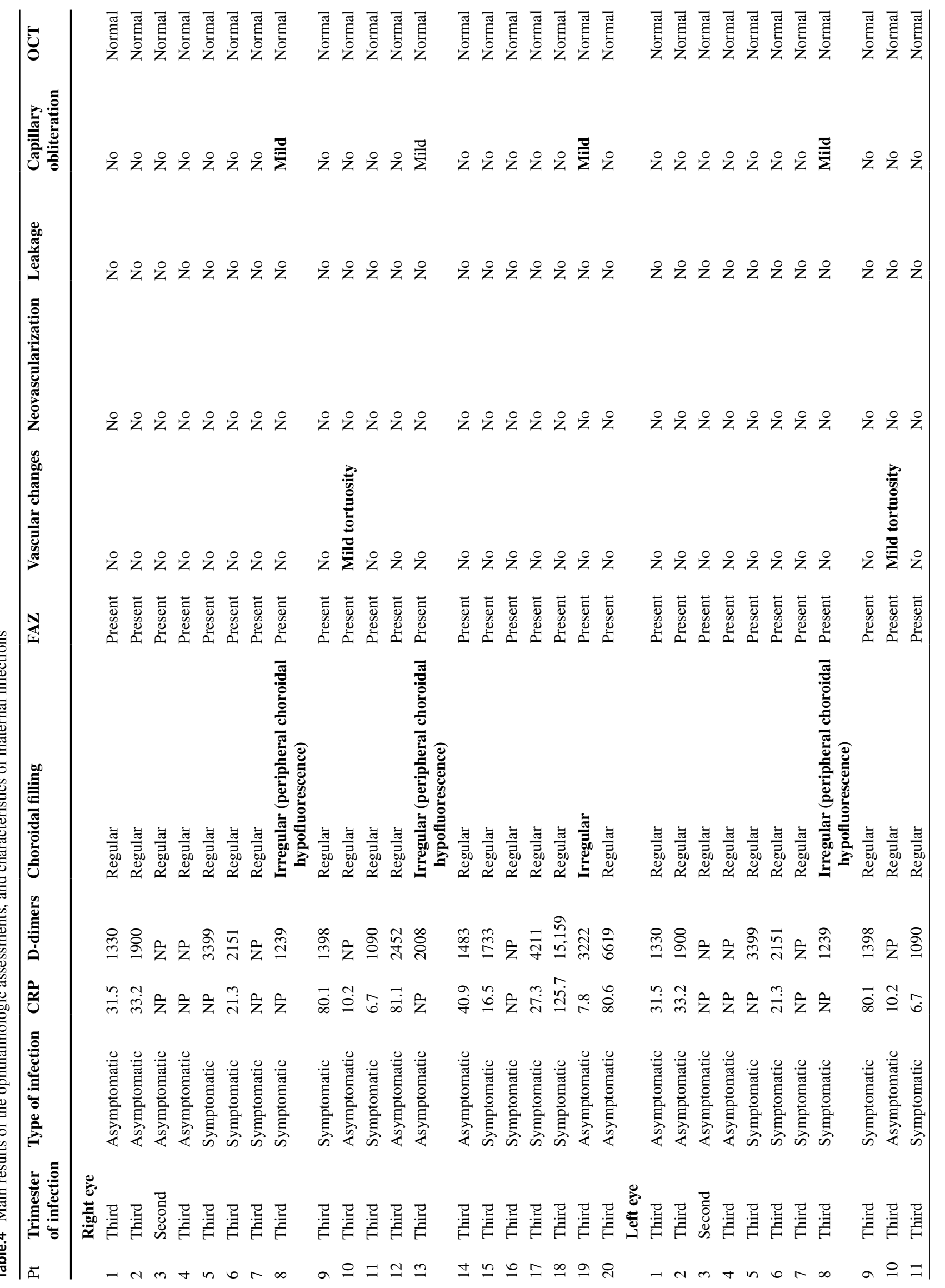




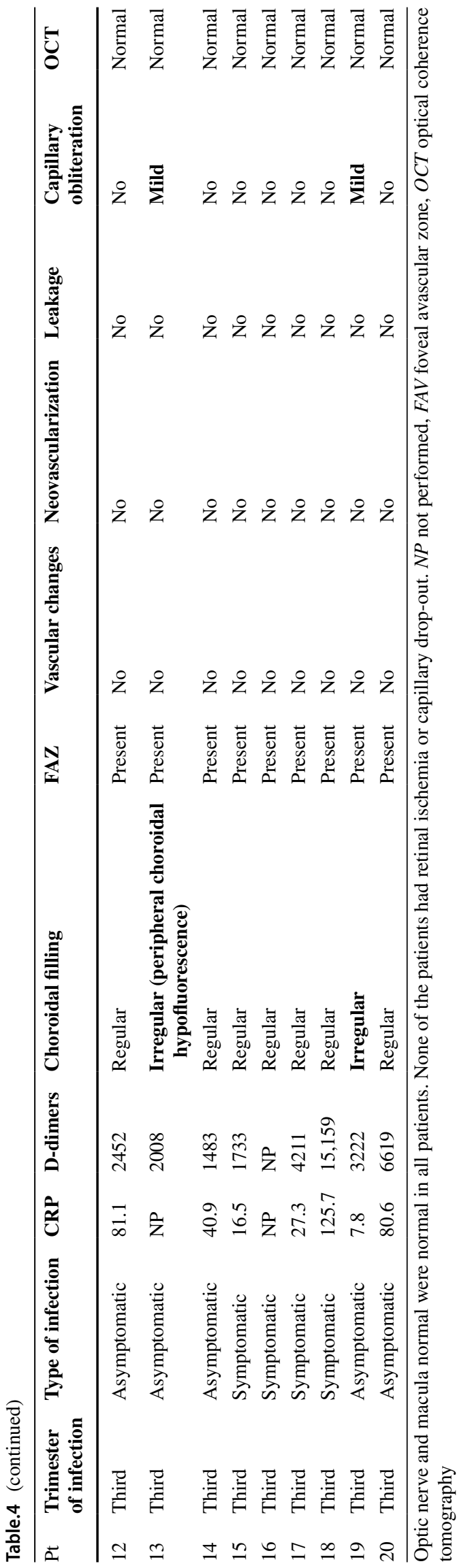

Visual acuity was normal in 15 tests out of $20(75 \%)$ and immature in the remaining 5 patients $(25 \%)$. Attention at distance was normal in 14 children $(70 \%)$ and reduced in the remaining 6 cases (30\%). The visual field was within normal limits in all children. Seventeen of the 20 evaluations performed reported normal contrast sensitivity, while it was reduced in $3(15 \%)$ infants.

The total score was lower than 2 (normal) in 17 of the 20 evaluations, equal to 2 (abnormal) in two evaluations and equal to 3 (abnormal) in the remaining case.

\section{Discussion}

In this study, we prospectively assessed the neonatal and postnatal multidisciplinary cohort of newborns exposed to SARS-CoV-2 during pregnancy or at time of delivery. Assessing a large proportion of pregnant women with documented SARS-CoV-2 infection, we found that only 4/171 newborns tested positive for SARS-CoV-2 at birth and there were no unfavorable neonatal outcomes in the neonatal period. Interestingly, all children enrolled so far at follow-up showed a normal general, auxological, neurological, and audiological follow-up, independently from the timing of maternal infection and the severity of COVID-19. Conversely, ophthalmologic outcomes, although preliminary since performed on only 21 children, showed abnormal findings in up to $15 \%$ of children.

The management of pregnant women and their newborns have been particularly challenging since the beginning of the pandemic. Although a first case series from China assessing nine pregnant women provided reassuring findings, showing that the clinical characteristics of COVID-19 pneumonia in pregnant women were similar to those reported for nonpregnant adult patients who developed COVID-19 pneumonia, and there was no vertical transmission of SARS-CoV-2 [24], the weak evidence led several clinicians to adopt a more conservative approach. In fact, Guillaume Favre and colleagues [25] soon suggested in their initial recommendations that "newborns of mothers positive for SARS-CoV-2 should be isolated for at least 14 days or until viral shedding clears, during which time direct breastfeeding is not recommended." Although these recommendations were not favorably universally welcomed since did not consider possible risks related to the mother-to-child separation and lack of breastfeeding [26], the previous experience with a new virus (Zika) suggested that when a new pathogen emerges, the healthcare community should be prepared for the worstcase scenario [27]. Consequently, given the uncertainties, most centers initially adopted a cautionary approach, as we also did in our center during the first wave of the pandemic. Later on, although the possibility of the vertical transmission have been demonstrated by De Luca and colleagues 
[3], further studies showed that this risk was low [5, 6], that breastfeeding did not increase the risk of an early postnatal infection, and that [4], overall, a conservative approach based on total mother-to-child separation and formula feeding was not justified.

In our center, since September 2021 all women with SARS-CoV-2 infection at delivery were allowed roomingin and breastfeed, with appropriate hand washing and masking. According to the recently established World Health Organization (WHO) guidelines [28], two children could be defined as having possible in utero SARS-CoV-2 transmission, while for the other two cases the in utero SARS-CoV-2 transmission was "indeterminate" since the second swabs were negative. In our routine practice we do not perform all possible procedures suggested by this guidance and we cannot exclude that some cases may have been missed. Also, we did not perform follow-up swabs and we cannot exclude horizontal postnatal asymptomatic infections in our samples, although none of these children developed signs or symptoms of infection. Anyway, our results are in line with previously published studies, which report a rate of vertical transmission ranging from 1 to $6 \%$ of deliveries [29-31]. However, Dumitri and colleagues found no evidence of vertical transmission in a 101 newborns tested in the USA [32].

While the neonatal outcomes are currently reassuring, the long-term effects on newborns exposed in utero or early in the postnatal life is still unknown and poorly addressed. The natural history of congenital infections taught us that even asymptomatic newborns can develop significant long-term effects in terms of audiologic, visual, and neuropsychiatric problems $[8,33,34]$. The recent evidence that maternal SARS-CoV-2 infection induces a robust placental inflammation [14] further reinforces this hypothesis, and for these reasons we decided, in our center, to offer a long-term multidisciplinary follow-up to all infants exposed in utero or within the first hours of life.

Importantly, we found that the auxological growth and neurological development was appropriate for all infants, independently by the severity of maternal disease. In particular, we purposely assessed if also the degree of inflammatory markers affected these parameters, and we did not find any significant relationships. This is a promising finding if we consider that, recently, infants exposed to Zika virus in utero could develop microcefalia and neuropsychiatric symptoms during follow-up [9].

During follow-up, we found that most infants did not present SARS-CoV-2 IgGs. This can be explained by several reasons. Most infections happened shortly before delivery, and therefore, there was not enough time to produce $\operatorname{IgG}$ and transfer them through the placenta; SARS-CoV-2 IgG less frequently cross the placenta, although a review found that about $90 \%$ of newborns born to positive mothers had IgG [35], false negative results, or rapid clearance of maternal
IgG when the child is not infected itself. The fact that a confirmed neonatal infection was significantly associated with positive IgG detected at 3 months of life can support the last hypothesis. However, a single $\operatorname{IgG}$ positivity at 3 months of age does not confirm vertical infection and we will need to continue follow-up to see if $\operatorname{IgG}$ levels will decrease or not. These hypotheses are also supported by Wang et al., which analyzed the seroconversion of 27 newborns born to 26 pregnant women infected with SARS-CoV-2. IgG transfer rates were $18.8 \%$ and $81.8 \%$ in those infants whose mother infected less and more than 2 weeks before delivery. In the first 2 months of life, the IgG level of infants dropped sharply to one tenth of that at birth [36]. These results and ours suggest that maternal SARS-CoV-2 IgG provides limited protection for infants due to rapid clearance.

Another important finding was that virtually all children had normal audiological outcomes, both at birth when screened with TEOAs and later when tested with ABR. However, our findings differed from those described by Celik et al., which reported, in a cohort of 37 infants exposed in utero at 36 healthy controls, an insufficiency in medial olivocochlear efferent system in infants exposed to SARSCoV-2 intrauterine [37]. Since in adults there are conflicting results concerning audiological outcomes of patients that recovered from SARS-CoV-2 infection [38], it is worth it to further address this issue in exposed infants as well.

Last, we assessed ophthalmological follow-up of a cohort of these children. Although results are still preliminary since only 20 children were assessed so far, ophthalmologic findings (Fig. 3) were less reassuring. About $15 \%$ of children had retinal abnormalities, such as bilateral choroidal perfusion anomalies, capillary obliteration, and bilateral vascular tortuosity. Conversely, behavioral assessment of visual function was mostly normal, except for the finding of reduced attention at distance in six cases (30\%) and reduced contrast sensitivity in three (15\%). So far, we found only one study assessing ophthalmology follow-up in 165 newborns exposed in utero [39]. Although SARSCoV-2 infected newborns did not present abnormalities, those exposed but not infected reported findings similar to ours, such as venous engorgement and vascular tortuosity and intraretinal hemorrhages. These data deserve further investigation, since the eye and its peculiar vascular structure may potentially represent a main target of indirect damage driven by placental inflammation related to SARSCoV-2 infection in pregnancy. However, since we did not include a control group or not-exposed children, we cannot provide firm conclusions on the meaning of these findings. Nevertheless, they deserve attention and further investigations since tests are already used in pediatric practice and also have a known prognostic role. In particular, in preterm infants with and without brain lesion, a normal visual assessment at term age is a good predictor of normal 
Fig. 3 Opthalmologic findings in newborns assessed by ultrawide-field fluorescein angiography (FFA). A and B Ultrawide-field FFA from patients 8 and 19 (see Table 4) showing mid-peripheral mild capillary obliteration and irregular vascular branching. $\mathbf{C}$ and D UW-FFA from patient 10 (see Table 4) showing mild bilateral arterial vascular tortuosity
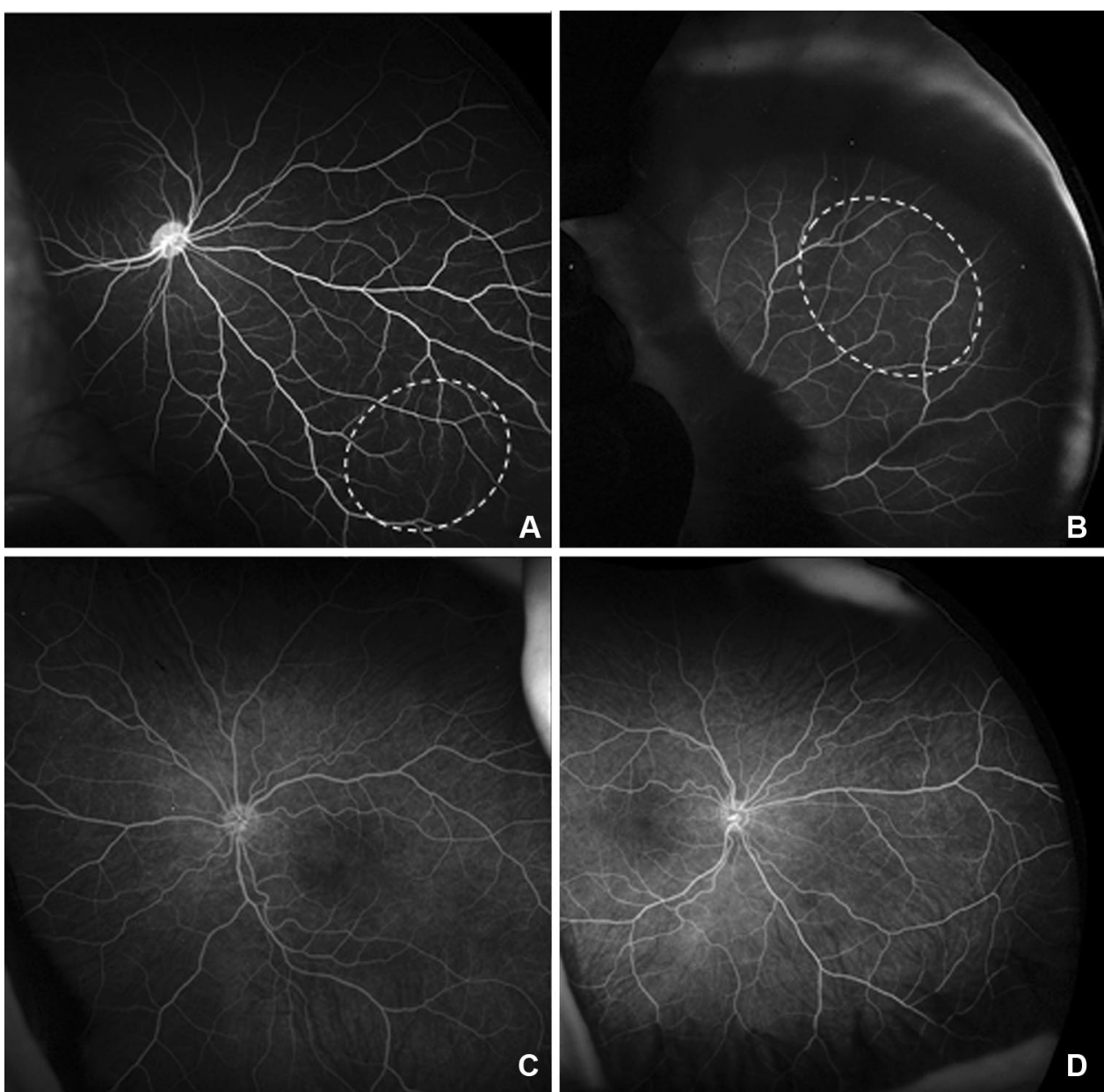

visual and neurodevelopmental outcome at 12 months [40]. The SD-OCT and FFA have already been performed for years on infants under 6 months of age with known retinal pathologies (retinopathy of prematurity, Coats disease, etc.). Several studies have highlighted the importance of SD-OCT imaging in detecting macular anomalies (macular edema, subretinal fluid, etc.) that are not visible on regular fundoscopic examination especially in neurovascular diseases like ROP [41]. Oral FFA has been proposed as an alternative to IV infusion of fluorescein that needs general anesthesia or sedation of the infants [42]. It has shown a valid method for follow-up imaging or detection of vascular anomalies.

Our study has limitations to address. The most important one is that the follow-up is still incomplete and that only a limited number of children completed the audiologic and ophthalmologic assessments. This is mainly because the large majority of infections in pregnant women happened during the second and third Italian waves (October-November and March-April). As a consequence, some children are still too young to enter some time points and, on the other hand, the high number of newborns born in a relatively short time, along with the massive pressure on our referral center and the need of strict precautions, only allowed us to perform a limited number of assessments per week. In addition, the lack of a control group requires taking with caution the results of the visual follow-up, which will need confirmation on larger samples. Last, the findings of our study mostly refer to neonates born from mothers affected by COVID-19 in the third trimester, since they represented the $93 \%$ of the study population. Therefore, our observations cannot be translated to those born from mothers infected on one earlier periods of pregnancy, which are those theoretically at higher risk for malformations.

However, due to the scarcity of data in this field, we felt that these preliminary data were still necessary to better inform other centers about the need of more data on longterm outcomes of these infants. Secondly, our findings are related to specific geographical area; therefore, ethnic differences that may affect inflammatory responses and, therefore, pathological changes could not be assessed.

In conclusion, our study showed that the neonatal and mid-term multidisciplinary outcomes of newborns exposed to SARS-CoV-2 infection in utero or during the first hours of life are mostly positive, except for ophthalmologic findings which, in a preliminary cohort, were abnormal in about 
$15 \%$ of cases. However, since our findings are preliminary, further prospective, longitudinal studies of cohorts followedup for years as happening with other infections acquired in pregnancy are necessary to better understand the clinical outcomes of children exposed to SARS-CoV-2 in utero and in the early postnatal life.

Supplementary Information The online version contains supplementary material available at https://doi.org/10.1007/s00431-021-04319-1.

Authors' contributions DB and PV conceptualized the study and wrote the first draft. SC, FP, GV, LG, and GZ were responsible for neonatal assessments. MSanguinetti and PC were responsible for microbiological assessments. MSali and DP were responsible for serologic assessments. ATC, SM, and MS were responsible for data collection. AA was responsible of statistical studies. GC, FC, AC, and BM were responsible for audiological studies. GM, LO, MP, and DR were responsible for ophthalmologic assessments. AL was responsible for maternal assessments. DR was responsible for neurological assessments. All authors read and approved the final version of the manuscript.

Data Availability Available upon request to the corresponding author.

\section{Declarations}

Ethics approval The study was approved by the Ethics Committee of the Fondazione Policlinico Universitario A. Gemelli IRCCS, Rome, Italy (ID 3104).

Consent to participate All patients' caregivers provided consent to participate to the study (MOD.PRO.122.001, version 1.1 FPG).

Consent for publication All patients' caregivers provided consent.

Conflict of interest The authors declare no competing interests.

\section{References}

1. Norman M, Navér L, Söderling J, Ahlberg M, Hervius Askling H, Aronsson B, Byström E, Jonsson J, Sengpiel V, Ludvigsson JF, Håkansson S, Stephansson O (2021) Association of maternal SARS-CoV-2 infection in pregnancy with neonatal outcomes. JAMA 325(20):2076-2086

2. Gale C, Quigley MA, Placzek A, Knight M, Ladhani S, Draper ES, Sharkey D, Doherty C, Mactier H, Kurinczuk JJ (2020) Characteristics and outcomes of neonatal SARS-CoV-2 infection in the UK: a prospective national cohort study using active surveillance. Lancet Child Adolesc Health S2352-4642(20):30342-30344

3. Vivanti AJ, Vauloup-Fellous C, Prevot S, Zupan V, Suffee C, Do Cao J, Benachi A, De Luca D (2020) Transplacental transmission of SARS-CoV-2 infection. Nat Commun 11(1):3572

4. Raschetti R, Vivanti AJ, Vauloup-Fellous C, Loi B, Benachi A, De Luca D (2020) Synthesis and systematic review of reported neonatal SARS-CoV-2 infections. Nat Commun 11(1):5164

5. Ronchi A, Pietrasanta C, Zavattoni M, Saruggia M, Schena F, Sinelli MT, Agosti M, Tzialla C, Varsalone FF, Testa L, Ballerini C, Ferrari S, Mangili G, Ventura ML, Perniciaro S, Spada E, Lunghi G, Piralla A, Baldanti F, Mosca F, Pugni L (2021) Evaluation of rooming-in practice for neonates born to mothers with severe acute respiratory syndrome coronavirus 2 infection in Italy. JAMA Pediatr 175(3):260-266
6. Salvatore CM, Han JY, Acker KP, Tiwari P, Jin J, Brandler M, Cangemi C, Gordon L, Parow A, DiPace J, DeLaMora P (2020) Neonatal management and outcomes during the COVID-19 pandemic: an observation cohort study. Lancet Child Adolesc Health 4(10):721-727. https://doi.org/10.1016/S2352-4642(20)30235-2

7. Penner J, Hernstadt H, Burns JE, Randell P, Lyall H (2021) Stop, think SCORTCH: rethinking the traditional "TORCH" screen in an era of re-emerging syphilis. Arch Dis Child 106(2):117-124

8. Turriziani Colonna A, Buonsenso D, Pata D, Salerno G, Chieffo DPR, Romeo DM, Faccia V, Conti G, Molle F, Baldascino A, De Waure C, Acampora A, Luciano R, Santangelo R, Valentini P (2020) Long-Term Clinical, Audiological, Visual, Neurocognitive and behavioral outcome in children with symptomatic and asymptomatic congenital cytomegalovirus infection treated with valganciclovir. Front Med (Lausanne) 24(7):268

9. Gazeta RE, Bertozzi APAP, Dezena RCAB, Silva ACB, Fajardo TCG, Catalan DT, Rizzo MFV, Moron AF, Soriano-Arandes A, Clemente NS, Quintella T, Ventura DF, Damico FM, França VCRM, Almeida JPG, Zara ALSA, Pires LC, Jundiaí CZV, Passos SD (2021) Three-year clinical follow-up of children intrauterine exposed to Zika virus. Viruses 13(3):523

10. Karimi-Galougahi M, Naeini AS, Raad N, Mikaniki N, Ghorbani $\mathbf{J}$ (2020) Vertigo and hearing loss during the COVID-19 pandemic — is there an association?. Acta Otorhinolaryngol Ital. https://doi. org/10.14639/0392-100X-N0820

11. Mao L, Jin H, Wang M et al (2020) Neurologic manifestations of hospitalized patients with coronavirus disease 2019 in Wuhan. China JAMA Neurol 77:683-690

12. Mustafa MWM (2020) Am J Otolaryngol Audiological profile of asymptomatic Covid-19 PCR positive cases. Am J Otolaryngol 41:102483

13. Kilic O, Kalcioglu MT, Cag Y et al (2020) Could sudden sensorineural hearing loss be the sole manifestation of COVID-19? An investigation into SARS-COV-2 in the etiology of sudden sensorineural hearing loss. Int J Infect Dis 97:208-211

14. Lu-Culligan A, Chavan AR, Vijayakumar P, Irshaid L, Courchaine EM, Milano KM, Tang Z, Pope SD, Song E, Vogels CBF, LuCulligan WJ, Campbell KH, Casanovas-Massana A, Bermejo S, Toothaker JM, Lee HJ, Liu F, Schulz W, Fournier J, Muenker MC, Moore AJ; Yale IMPACT Team, Konnikova L, Neugebauer KM, Ring A, Grubaugh ND, Ko AI, Morotti R, Guller S, Kliman HJ, Iwasaki A, Farhadian SF (2021) Maternal respiratory SARS-CoV-2 infection in pregnancy is associated with a robust inflammatory response at the maternal-fetal interface. Med (N Y) 2(5):591-610. e10. https://doi.org/10.1016/j.medj.2021.04.016. Epub 2021 Apr 30

15. Gorga M, Neely S, Ohlrich B, Hoover B, Redner J, Peters J (1997) From laboratory to clinic: a large scale study of distortion product otoacoustic emissions in ears with normal hearing and ears with hearing loss. Ear Hear 18(6):440-455

16. Hussain D, Gorga M, Neely S, Keefe D, Peters J (1998) Transient evoked otoacoustic emissions in patients with normal hearing and in patients with hearing loss. Ear Hear 19(6):434-449

17. Recommended protocol for performing oral fundus fluorescein angiography (FFA) in children. Oliver R Marmoy et al. Eye (Lond) 2020 Dec 15

18. Maldonado RS et al (2010) Optimizing hand-held spectral domain optical coherence tomography imaging for neonates, infants, and children. Investig Ophthalmol Vis Sci 51:2678-2685

19. Mercuri E, Baranello G, Romeo DM, Cesarini L, Ricci D (2007) The development of vision. Early Hum Dev 83:795-800

20. Ricci D, Romeo DMM, Serrao F, Cesarini L, Gallini F, Cota F, Zuppa A, Romagnoli C, Cowan F, Mercuri E (2008) Application of a neonatal assessment of visual function in a population of low risk full-term newborn. Early Hum Dev 84:277-280

21. Ricci D, Cesarini L, Romeo DM, Gallini F, Serrao F, Groppo M, De Carli A, Cota F, Lepore D, Molle F, Ratiglia R, De 
Carolis MP, Mosca F, Romagnoli C, Guzzetta F, Cowan F, Ramenghi LA, Mercuri E (2008) Visual function at 35 and 40 weeks' postmenstrual age in low-risk preterm infants. Pediatrics 122:e1193-e1198

22. Ricci D, Cesarini L, Gallini F, Serrao F, Leone D, Baranello G, Cota F, Pane M, Brogna C, De Rose P, Vasco G, Alfieri P, Staccioli S, Romeo DM, Tinelli F, Molle F, Lepore D, Baldascino A, Ramenghi LA, Torrioli MG, Romagnoli C, Cowan F, Atkinson J, Cioni G, Mercuri E (2010) Cortical visual function in preterm infants in the first year. J Pediatr 156:550-555

23. Zeng L, Xia S, Yuan W, Yan K, Xiao F, Shao J, Zhou W (2020) Neonatal early-onset infection with SARS-CoV-2 in 33 neonates born to mothers with COVID-19 in Wuhan. China JAMA Pediatr 174(7):722-725

24. Chen H, Guo J, Wang C, Luo F, Yu X, Zhang W, Li J, Zhao D, Xu D, Gong Q, Liao J, Yang H, Hou W, Zhang Y (2020) Clinical characteristics and intrauterine vertical transmission potential of COVID-19 infection in nine pregnant women: a retrospective review of medical records. Lancet 395(10226):809-815. https:// doi.org/10.1016/S0140-6736(20)30360-3. Epub 2020 Feb 12. Erratum in: Lancet. 2020 Mar 28;395(10229):1038

25. Favre G, Pomar L, Qi X, Nielsen-Saines K, Musso D, Baud D (2020) Guidelines for pregnant women with suspected SARSCoV-2 infection. Lancet Infect Dis 20(6):652-653. https://doi. org/10.1016/S1473-3099(20)30157-2. Epub 2020 Mar 3. PMID: 32142639; PMCID: PMC7134390

26. Schmid MB, Fontijn J, Ochsenbein-Kölble N, Berger C, Bassler D (2020) COVID-19 in pregnant women. Lancet Infect Dis 20(6):653. https://doi.org/10.1016/S1473-3099(20)30175-4. Epub 2020 Mar 17. PMID: 32197098; PMCID: PMC7270341

27. Musso D, Ko AI, Baud D (2019) Zika virus infection - after the pandemic. N Engl J Med 381(15):1444-1457. https://doi.org/10. 1056/NEJMra1808246 (PMID: 31597021)

28. World Health Organization (2021) Definition and categorization of the timing of mother-to-child transmission of SARS-CoV-2: scientific brief, 8 February 2021. World Health Organization. https://apps.who.int/iris/handle/10665/339422. License: CC BY-NC-SA 3.0 IGO

29. Tolu LB, Ezeh A, Feyissa GT (2021) Vertical transmission of severe acute respiratory syndrome coronavirus 2: a scoping review. PLoS One 16(4):e0250196

30. Shah PS, Diambomba Y, Acharya G, Morris SK, Bitnun A (2020) Classification system and case definition for SARS-CoV-2 infection in pregnant women, fetuses, and neonates. Acta Obstet Gynecol Scand 99(5):565-568

31. Flaherman VJ, Afshar Y, Boscardin J, Keller RL, Mardy A, Prahl MK, Phillips C, Asiodu IV, Berghella WV, Chambers BD, CrearPerry J, Jamieson DJ, Jacoby VL, Gaw SL (2020) Infant outcomes following maternal infection with SARS-CoV-2: first report from the PRIORITY study. Clin Infect Dis 18:ciaa1411

32. Dumitriu D, Emeruwa UN, Hanft E, Liao GV, Ludwig E, Walzer L, Arditi B, Saslaw M, Andrikopoulou M, Scripps T, Baptiste C, Khan A, Breslin N, Rubenstein D, Simpson LL, Kyle MH, Friedman AM, Hirsch DS, Miller RS, Fernández CR, Fuchs KM, Keown MK, Glassman ME, Stephens A, Gupta A, Sultan S, Sibblies C, Whittier S, Abreu W, Akita F, Penn A, D'Alton ME, Orange JS, Goffman D, Saiman L, Stockwell MS, GyamfiBannerman C (2021) Outcomes of neonates born to mothers with severe acute respiratory syndrome coronavirus 2 infection at a large medical center in New York City. JAMA Pediatr 175(2):157-167. https://doi.org/10.1001/jamapediatrics.2020. 4298.PMID:33044493;PMCID:PMC7551222

33. Gaur P, Ffrench-Constant S, Kachramanoglou C, Lyall H, Jan W (2020) Is it not time for international guidelines to combat congenital cytomegalovirus infection? A review of central nervous system manifestations. Clin Radiol 75(8):644.e7-644.e16. https:// doi.org/10.1016/j.crad.2020.02.009 (Epub 2020 Mar 23 PMID: 32216960)

34. McCarthy J, Liu D, Kaskel F (2021) The need for life-course study of children born to mothers with prior COVID-19 infection. JAMA Pediatr. https://doi.org/10.1001/jamapediatrics.2021.2423. Epub ahead of print. PMID: 34279556

35. Bwire GM, Njiro BJ, Mwakawanga DL, Sabas D, Sunguya BF (2021) Possible vertical transmission and antibodies against SARS-CoV-2 among infants born to mothers with COVID-19: a living systematic review. J Med Virol 93(3):1361-1369

36. Wang X, Yang P, Zheng J, Liu P, Wei C, Guo J, Zhang Y, Zhao D (2021) Dynamic changes of acquired maternal SARS-CoV-2 IgG in infants. Sci Rep 11(1):8021

37. Celik T, Simsek A, Koca CF, Aydin S, Yasar S (2021) Evaluation of cochlear functions in infants exposed to SARS-CoV-2 intrauterine. Am J Otolaryngol 42(4):102982. https://doi.org/10.1016/j. amjoto.2021.102982. Epub 2021 Feb 18. PMID: 33621767; PMCID: PMC7890354

38. Dror AA, Kassis-Karayanni N, Oved A, Daoud A, Eisenbach N, Mizrachi M, Rayan D, Francis S, Layous E, Gutkovich YE, Taiber S, Srouji S, Chordekar S, Goldenstein S, Ziv Y, Ronen O, Gruber M, Avraham KB, Sela E (2021) Auditory performance in recovered SARS-COV-2 patients. Otol Neurotol 42(5):666-670. https://doi. org/10.1097/MAO.0000000000003037.PMID:33967243;PMCID: PMC8115428

39. Kiappe OP, Santos da Cruz NF, Rosa PAC, Arrais L, Bueno de Moraes NS (2021) Ocular assessments of a series of newborns gestationally exposed to maternal COVID-19 infection. JAMA Ophthalmol 7:e211088. https://doi.org/10.1001/jamaophthalmol. 2021.1088. Epub ahead of print. PMID: 33825821; PMCID: PMC8027939

40. Ricci D, Romeo DM, Gallini F, Groppo M, Cesarini L, Pisoni S, Serrao F, Papacci P, Contaldo I, Perrino F, Brogna C, Bianco F, Baranello G, Sacco A, Ometto A, Cilauro S, Mosca F, Romagnoli C, Romeo MG, Cowan F, Cioni G, Ramenghi LA, Mercuri E (2011) Early visual assessment in preterm infants with and without brain lesions: correlation with visual and neurodevelopmental outcome at 12 months. Early Hum Dev 87:177-182

41. Maldonado RS, O'Connell RV, Sarin N, Freedman SF, Wallace DK, Cotten CM, Winter KP, Stinnett S, Chiu SJ, Izatt JA, Farsiu S, Toth CA (2011) Dynamics of human foveal development after premature birth. Ophthalmology 118(12):2315-2325

42. Fung TH, Muqit MM, Mordant DJ, Smith LM, Patel CK (2014) Noncontact high-resolution ultra-wide-field oral fluorescein angiography in premature infants with retinopathy of prematurity. JAMA Ophthalmol 132(1):108-110

Publisher's Note Springer Nature remains neutral with regard to jurisdictional claims in published maps and institutional affiliations. 


\section{Authors and Affiliations}

\section{Danilo Buonsenso ${ }^{1,2,3}$ [D - Simonetta Costa ${ }^{1}$. Lucia Giordano ${ }^{1} \cdot$ Francessca Priolo ${ }^{1}$. Arianna Turriziani Colonna' Sofia Morini ${ }^{1}$. Martina Sbarbati ${ }^{1}$. Davide Pata ${ }^{1}$. Anna Acampora ${ }^{1}$. Guido Conti ${ }^{4}$. Fabrizio Crudo ${ }^{4}$. Alessandro Cantiani ${ }^{4}$. Bianca Maria Martina ${ }^{4}$. Giulia Maria Amorelli ${ }^{5,6}$. Lorenzo Orazi ${ }^{6} \cdot$ Maria Petrianni $^{6}$. Daniela Ricci $^{6}$. Antonio Lanzone ${ }^{1}$ - Maurizio Sanguinetti ${ }^{3,7}$. Paola Cattani ${ }^{3,7} \cdot$ Michela Sali $^{3,7}$. Domenico Romeo ${ }^{1}$. Giuseppe Zampino ${ }^{1} \cdot$ Giovanni Vento $^{1} \cdot$ Piero Valentini ${ }^{1}$}

Danilo Buonsenso

danilo.buonsenso@policlinicogemelli.it;

danilobuonsenso@gmail.com

Simonetta Costa

simonetta.costa@policlinicogemelli.it

Lucia Giordano

lucia.giordano@policlinicogemelli.it

Francessca Priolo

francesca.priolo@policlinicogemelli.it

Arianna Turriziani Colonna

arianna.turrizianic@gmail.com

Sofia Morini

sofia.morini@gmail.com

Martina Sbarbati

martina.sbarbati@outlook.it

Davide Pata

davide.pata01@gmail.com

Anna Acampora

anna.acampora@unicatt.it

Guido Conti

guido.conti@unicatt.it

Fabrizio Crudo

fabriziocrudo@virgilio.it

Alessandro Cantiani

cantiani.ale@outlook.it

Bianca Maria Martina

biancamaria.martina@unicatt.it

Giulia Maria Amorelli

amorelligiulia@gmail.com

Lorenzo Orazi

Lorenzo.orazi@policlinicogemelli.it

Maria Petrianni

maria.petrianni@policlinicogemelli.it

Antonio Lanzone

antonio.lanzone@unicatt.it
Maurizio Sanguinetti

maurizio.sanguinetti@unicatt.it

Paola Cattani

paola.cattani@policlinicogemelli.it

Michela Sali

michela.sali@unicatt.it

Domenico Romeo

domenico.romeo@policlinicogemelli.it

Giuseppe Zampino

giuseppe.zampino@unicatt.it

Giovanni Vento

giovanni.vento@unicatt.it

Piero Valentini

piero.valentini@unicatt.it

1 Department of Woman and Child Health and Public Health, Fondazione Policlinico Universitario A Gemelli IRCCS,

Rome, Italy

2 Global Health Research Institute, Istituto di Igiene, Università Cattolica del Sacro Cuore, Rome, Italy

3 Dipartimento di Scienze Biotecnologiche di Base, Cliniche Intensivologiche e Perioperatorie, Sezione di Microbiologia, Università Cattolica del Sacro Cuore, Rome, Italy

4 Institute of Otorhinolaryngology, Università Cattolica del Sacro Cuore, Fondazione Policlinico "A Gemelli," IRCCS, Rome, Italy

5 Department of Ophthalmology, Gemelli Foundation IRCSS, Catholic University of the Sacred Heart, Rome, Italy

6 National Centre of Services and Research for the Prevention of Blindness and Rehabilitation of Low Vision Patients, IAPB Italia Onlus- Fondazione Policlinico Agostino Gemelli IRCCS, Rome, Italy

Dipartimento di Scienze di Laboratorio E Infettivologiche, Fondazione Policlinico Universitario A Gemelli IRCCS, Rome, Italy 